\title{
Translational genomics for human diseases: toward a new era of precision medicine
}

\author{
Yoon Shin $\mathrm{Cho}^{1} \cdot \mathrm{Ki}$ Wha Chung ${ }^{2} \cdot \mathrm{Nam}-\mathrm{Soo} \mathrm{Kim}^{3}$
}

Received: 15 May 2016/Accepted: 22 May 2016/Published online: 31 May 2016

(C) The Genetics Society of Korea and Springer-Science and Media 2016

Our understanding of ourselves has greatly expanded since the first genome sequence was released 25 years ago. However, our genome still presents many challenges to comprehending the problems related to human diseases. The Precision Medicine Initiative announced by US President Barack Obama in the State of the Union Address on January 20, 2015 is a bold new research effort aiming to customize healthcare with medical decisions, practices, and/or products tailored to the individual patient. Considering the major goals of precision medicine-to improve human health and treat disease-genetics and genomics can play important roles in identifying the genetic basis of diseases, elucidating the underlying biological mechanisms in the development of diseases, and discovering genetic factors for individual drug responsiveness. From that point of view, Genes \& Genomics intended to provide a forum for discussing precision medicine-related topics and invited articles and reviews for a special issue on the theme of "Translational Genomics for Human Diseases". The editorial board carefully screened a number of papers submitted to the journal. A total of eight research articles and one review have been selected for the intended special issue. We hope that the special issue provides our readers with an update and overview of the development of

Nam-Soo Kim

kimnamsu@kangwon.ac.kr

1 Department of Biomedical Science, Hallym University, Chuncheon, Korea

2 Department of Biological Sciences, Kongju National University, Gongju, Korea

3 Department of Molecular Bioscience, Institute of Bioscience and Biotechnology, Kangwon National University, Chuncheon, Korea concepts, technologies, and applications of the humangenome database to help overcome human diseases and meet the goals of precision medicine.

One of the major surprising findings from the human genome is that the number of coding genes is far less than what we originally thought. It was hardly believable that only about 20,700 genes can account for the human complexity from the view of the early classical dogma of "onegene-one-protein", which was oversimplified (Consortium 2012). We now know that the actual gene-protein relationship is much more complex, often being viewed as "onegene-many-proteins" or "one-gene-many-functions". The expansion of functions of single genes is provided mainly through alternate splicing (AS). Indeed, AS affects more than $86 \%$ of human genes. Uversky (2016) summarized the effects of aberrations in AS on some key, intrinsically disordered proteins and the pathogenesis of neurodegenerative diseases, cardiovascular disease, cancer, and diabetes. With extensive reviews on the relation between AS and human diseases, he suggests that understanding of the molecular mechanisms underlying the modulation of the biological activities of numerous spliced variants contributes to new drug discovery.

Of the many diabetes mellitus-associated complications, diabetic nephropathy (DN) is the most challenging. DN affects $\sim 30 \%$ of patients with long-standing diabetes, and the prevalence of DN is increasing continuously, posing a major challenge to the health-care system in modern society (Ahn et al. 2014). One research article was included in this special issue on the functional mechanisms of DNassociated genetic variants (Gong et al. 2016). Gong and colleagues reported two single-nucleotide polymorphisms (SNPs) (rs3135377 and rs 9469220) that act as cis-effect regulators of the gene identified for DN by conducting integrative analyses (comprising expression analysis of 
quantitative-trait loci, differential gene-expression analysis, and functional prediction analysis) using a publicly available dataset. The authors also predict that those two SNPs might act as regulatory sites based on online prediction tools. Their findings provide potential mechanistic links underlying the association between DN and the two identified SNPs.

Another research article in this issue is on chronic kidney disease. Lim et al. (2016) reported on a genetic-association study to detect genetic loci for kidney functionrelated traits such as blood urea nitrogen (BUN) and blood albumin levels in subjects stratified by diabetic status. Their genome-wide association analyses detected one locus, LOC105374266, showing a significant association with BUN in nondiabetic normal subjects. That locus might provide new insight into genetic predisposition to chronic kidney disease in human populations. They also extended their association studies between kidney function-related traits and three microRNA variants from which microRNA-related SNPs were selected from two population cohorts. Their findings provide a clue of the functional role of microRNA in the development of chronic kidney disease in human populations.

Mesenchymal stem cells (MSCs) and stromal cells derived from MSCs have been recognized as key players in carcinogenesis, affecting tumor growth, development, and progression during the early stages of tumorogenesis. It was known that those cells can influence the construction of the microenvironment, epithelial mesenchymal transit, and metastasis (Calon et al. 2015). A research article in this issue deals with DNA methylation changes in the genes in the extracellular matrix (ECM) remodelling pathway during the transformation of human MSCs (Kim et al. 2016a). The ECM provides physical support for tissue specificity and elasticity in the form of basement membrane. It is also a dynamic structure that is constantly remodeled to control tissue homeostasis. The authors found that promoter hypermethylation was correlated with transcription silencing in three ECM genes (LAMA1, FBN2, and IGFBP3) during the transformation of an MSC model system in human colon cancer-cell lines. Their findings suggest that epigenetic alterations in ECM-pathway genes might contribute to carcinogenesis.

Spinal cord injury (SCI) is a devastating neurological disorder. Because it accompanies long-lasting disability and limited response to acute drug administration and efforts at rehabilitation, it impacts enormously on the quality of life and economy of patients. It is commonly estimated that more than 2.5 million people suffer from SCI, and more than 130,000 new cases are reported worldwide each year (McDonald and Sadowsky 2002). In this issue, Peng et al. (2016) analyzed the potential pathways and target genes in SCI using bioinformatic approaches. They reported 173 overlapping, differentially expressed genes (DEGs) between raphe-magnus and sensorimotor-cortex samples by analyzing publicly available microarray data. Findings from their pathway-enrichment analysis indicate that overlapping DEGs in SCI are mainly related to inflammation and immunity. They identified several key genes (RAC2, CD44, and $A P R C 1 B)$ that impact on the pathogenesis of SCI by participating in different pathways. The bioinformatic approaches revealed potential pathways and target genes in SCI, however, the results obtained from their bioinformatic methods need further experimental studies to verify the findings.

Alzheimer's disease (AD) is a neurodegenerative disease accounting for $60-70 \%$ of cases of dementia (Burns and Lliffe 2009). It usually starts slowly and gets worse over time until cognitive abilities are completely lost. Using AD model mice, Lee and Choe (2016) reported region-specific changes in the distribution of transient receptor potential vanilloid 4 channel (TRPV4) in the central nervous system. They found that TRPV4 was upregulated in the cerebral cortex, hippocampal formation, striatum, and thalamus of early-stage and late-stage $\mathrm{AD}$ model mice compared with that in control mice. They also found increased expression of TRPV4 in the hippocampal formation, striatum, and thalamus of late-stage AD model mice, which was the first demonstration of AD-related increases in TRPV4 expression in the brain. Their findings provide a useful clue to understanding the development of AD-related neurodegenerative diseases. Understanding the functional significance of the regulation of TRPV4 in AD will require further research.

Edwards' syndrome (ES) is also known as trisomy 18. Its frequency is about 1 in 6000 individuals, of which $80 \%$ are female (http://www.trisomy 18.org/what-is-trisomy-18/). ES fetuses are prone to miscarrige, with an abortion rate of 72-87 \% (Lakovschek et al. 2011). When abortion does not occur, babies are highly underdeveloped and die within 1 year of birth (Sibiude et al. 2011). Li et al. (2016) present an article in this issue on the use of three short tandem repeat (STR) loci (D18S53,D18S59, and D18S488) in the diagnosis of ES. For that purpose, they used quantitative fluorescence PCR. The technique was highly sensitive, specific, simple, and rapid. Their report suggests clinical applications of the three STRs to the large-scale genetic and prenatal diagnosis of ES.

Defensins are cystein-rich cationic proteins 19-45 amino acids in length found in both vertebrates and invertebrates as well as in plants. They are active agents against pathogenic bacteria, fungi, and some viruses (Lehrer 2004). Human beta-defensins play a crucial role in innate immune responses, acting at the initial stages of immune defense against microbial infections. An article in this issue reports on genetic associations between $D E F B 1$ 
polymorphisms and chronic respiratory diseases (CRDs) based on a meta-analysis comprising 1343 CRD cases and 1261 healthy controls (Ran et al. 2016). Two SNPs showed strong evidence of association with CRD susceptibility. Those findings imply the functional relevance of $D E F B 1$ to the development of CRDs.

Charcot-Marie-Tooth (CMT) disease is a genetically and clinically heterogeneous disorder that is associated with more than 80 causative genes (Rossor et al. 2013). Kim et al. (2016b) report in this issue on the genotypephenotype correlation of CMT type 1E patients with peripheral myelin protein 22 (PMP22) mutations. For their analysis, they performed whole-exome sequencing, histological examination of the distal sural nerves, and magnetic resonance imaging of the legs of two patients. They found two de novo mutations in PMP22 in both patients. They found that two missense mutations are correlated with the patients' numerous characteristic phenotypes. Their findings provide a clue to understanding the genetic causes of CMT and a way to approach CMT diagnosis.

\section{References}

Ahn JH, Yu JH, Ko SH, Kwon HS, Kim DJ, Kim JH, Kim CS, Song KH, Won JC, Lim S et al (2014) Prevalence and determination of diabetic nephropathy in Korea: Korea National Health and Nutrition Survey. Diabetes Metab J 38:109-119

Burns A, Lliffe S (2009) Alzheimer's disease. BMJ. doi:10.1136/bmj.b158

Calon A, Lonardo E, Berenguer-Llergo A, Espinet E, HernandoMomblona $\mathrm{X}$, Iglesias $\mathrm{M}$, Sevillano $\mathrm{M}$, Palomo-Ponce S, Tauriello DVF, Byrom D et al (2015) Nat Genet 47:320-329

Consortium TEP (2012) An integrated encyclopedia of DNA elements in the human genome. Nature 489:57-74

Gong C, Xu Y, Fan Y, Liu X, Xiong C, He L, Liu C, Rao S, Xia W, Ding L et al (2016) Functional mechanisms for diabetic nephropathy-associated genetic variants. Genes Genom. doi:10. 1007/s13258-016-0415-5
Kim TO, Park SH, Kim HS, Ahuja N, Yi JM (2016a) DNA methylation changes in extracellular remodelling pathway genes during the transformation of human mesenchymal stem cells. Genes Genom. doi:10.1007/s13258-016-0402-X

Kim JY, Koo HS, Park KD, Choi SS, Yu JS, Hong YB, Chung KW, Choi BO (2016b) Genotype-phenotype correlation of CharcotMarie-Tooth type 1E patients with PMP22 mutations. Genes Genom. doi:10.1007/s13258-016-0423-5

Lakovschek IC, Streubel B, Ulm B (2011) Natural outcome of trisomy 13 , trisomy 18 , and triploidy after prenatal diagnosis. Am J Med Genet A 155A:2626-2633

Lee JC, Choe SY (2016) Region-specific changes in the distribution of transient receptor potential vanilloid 4 channel (TRPV4) in the central nervous system of Alzheimer's disease model. Genes Genom. doi:10.1007/s13258-016-0389-3

Lehrer R (2004) Primate defensins. Nat Rev Microbiol 2:727-738

Li X, Sun L, Shi Y, Ju D, Li Y, Zhang Y, Yue T (2016) Use of the STR D18S53, D18S59, and D18S488 in the diagnosis of Edwards' syndrome. Genes Genom. doi:10.1007/s13258-0160412-8

Lim JS, Koh IS, Cho YS (2016) Identification of genetic loci stratified by diabetic status and microRNA related SNPs influencing kidney function in Korean Population. Genes Genom. doi:10. 1007/s13258-016-0411-9

McDonald JW, Sadowsky C (2002) Spinal-cord injury. Lancet 359:417-425

Peng D, Chen M, Zuo G, Shan S, Gao C, Zhao G (2016) Analysis of the potential pathways and target genes in spinal cord injury using bioinformatics methods. Genes Genom. doi:10.1007/s13258-0160385-7

Ran X, Chen J, Chen L, Li Y, Wu M (2016) Beta-defensin gene (DEFB1) polymorphisms are associated with the susceptibility to chronic respiratory diseases. Gene Genom. doi:10.1007/s13258016-0388-4

Rossor AM, Polke JM, Houlden H, Reilly MM (2013) Clinical implications of genetic advances in Chrie-Marie-Tooth disease. Nat Rev Neurol 9:562-571

Sibiude J, Gavard L, Floch-Tudal C, Madelbrot L (2011) Prenatal care and outcome of fetuses with trisomy 13 and 18 following a prenatal decision not to terminate the pregancy. Fetal Diagn Ther 29:233-237

Uversky VN (2016) (Intrinsically disordered) splice variants in the proteome: implications for novel drug delivery. Genes Genom. doi:10.1007/s13258-015-0384-0 